\section{Tabagismo, situação no mercado de trabalho e gênero: análise da PNAD 2008}

\author{
Smoking, work status, and gender: analysis of the \\ Brazilian National Household Sample Survey, 2008
}

Luana Giatti 1

Sandhi Maria Barreto 1

\section{Introdução}

This study investigated whether men's and women's work status is associated with current smoking, after adjusting for schooling, income, and health, besides discussing hypotheses for such associations. The sample included individuals 15 to 64 years of age living in metropolitan areas, currently in the work force, and interviewed in the Brazilian National Household Sample Survey (PNAD) in 2008. Prevalence ratios (PR) and 95\% confidence intervals (95\%CI) were obtained by Poisson regression. After adjusting, smoking prevalence was statistically higher in individuals with informal jobs (men, $P R=1.31$, 95\%CI: 1.24-1.38; women, $P R=1.22$, 95\%CI: 1.12-1.31) or who were unemployed (men, $P R=1.31$, 95\%CI: 1.18-1.44; women, $P R=1.16$, 95\%CI: 1.03-1.32). Our results confirm that work status is an independent dimension of social inequalities in smoking. The social gradient was steeper in men, but the association between work status and smoking was present in both genders

Smoking; Job Market; Health Inequalities
Evidências científicas acerca dos efeitos deletérios da exposição ao tabaco sobre a saúde vêm se acumulando desde a década de 1950. Entre esses efeitos, destacam-se as doenças cardiovasculares e respiratórias, cânceres, doenças mentais, incapacidades e morte ${ }^{1}$.

Medidas restritivas na comercialização e consumo do tabaco foram adotadas em vários países, com consequente queda do tabagismo principalmente nos países considerados mais ricos 2 . No entanto, mesmo nestes, o declínio do consumo não ocorre de maneira uniforme entre os diferentes grupos sociais. Os estudos sugerem gradiente inverso entre indicadores de posição socioeconômica e prevalência de tabagismo ${ }^{3}$. Ser fumante é crescentemente associado a diferentes marcadores de privação, como escolaridade, renda e ocupação dos indivíduos, condições materiais do domicílio 4 e fatores contextuais relativos à vizinhança 5 . O tabagismo tende a aumentar diretamente com o nível de privação entre adultos mais jovens e mais velhos, homens e mulheres, na vida produtiva e na aposentadoria 6 .

Na França, entre 2000 e 2007, observou-se queda diferencial do consumo de tabaco segundo indicadores de nível socioeconômico 7 . $\mathrm{Na}$ Itália, em 2008, foram registradas as menores taxas de prevalência de tabagismo dos últimos cinquenta anos, e estas foram ainda mais baixas entre os indivíduos de maior escolaridade 8 . Nos 
Estados Unidos, dados do National Health Interview Survey do ano de 2000 identificaram que o tabagismo era mais frequente entre os indivíduos com menor escolaridade, com menor renda e em ocupações menos qualificadas ${ }^{9}$. Estudo realizado na Austrália apontou queda mais acentuada do tabagismo entre indivíduos de maior nível socioeconômico, embora a análise por gênero tenha mostrado que essa desigualdade ocorria apenas entre os homens 10.

No Brasil, também há tendência de queda da prevalência de fumantes. A proporção de fumantes na população adulta brasileira passou de $35 \%$ em 1998 para 22\% em 2003, declínio mais acentuado entre os homens 11 . Como em outros países, a prevalência de tabagismo tende a ser maior entre os indivíduos em desvantagens socioeconômicas. Dados referentes a 16 capitais brasileiras e ao Distrito Federal, obtidos em 2002 e 2003, mostraram prevalências mais acentuadas nos grupos com menor escolaridade 12 . Inquérito conduzido em todas as capitais no ano de 2006 identificou maiores prevalências entre os indivíduos de baixa escolaridade, especialmente entre os mais jovens (18 a 29 anos) 13. O declínio nas taxas de prevalência entre 1998 e 2003 foi diretamente relacionado com o poder aquisitivo das famílias 11.

Se, no início da expansão do tabagismo, as pessoas de nível socioeconômico mais alto apresentavam maior consumo, a tendência atual é maior exposição entre as mais pobres 1 . A inserção no mercado de trabalho expressa uma dimensão das desigualdades sociais em saúde. Os estudos têm fornecido evidências de que o trabalho e a ocupação contribuem para o gradiente da associação inversa entre inserção social e saúde. Hierarquia ocupacional, estressores psicossociais, condições de trabalho ou perda do emprego são alguns dos mecanismos por meio dos quais o trabalho pode contribuir com esse gradiente 14 . Em decorrência das mudanças socioeconômicas estruturais ocorridas no final do século passado, o impacto das formas de trabalho precárias ganhou relevância na investigação da relação trabalho e saúde. Em geral, essas ocupações relacionam-se a piores condições e ambientes ocupacionais, a instabilidade, a menores rendimentos, garantias e suportes sociais. Além disso, esses trabalhadores estão sob maior risco de desemprego 15. Por essas razões, indivíduos com inserções precárias no mercado de trabalho tendem a apresentar piores indicadores sociais e de qualidade de vida do que trabalhadores em empregos formais.

Considerando que inserção no trabalho, ainda que relacionada com escolaridade e renda, é uma expressão distinta de condição social, é possível que a prevalência e a tendência de queda do tabagismo variem segundo a inserção dos indivíduos no trabalho. Espera-se que o tabagismo atual seja mais frequente entre aqueles que estão desempregados ou em condições de trabalho mais precárias, quando comparados aos trabalhadores com empregos formais, independentemente da escolaridade e da renda. É possível também que, assim como a associação entre inserção no trabalho e saúde difere entre homens e mulheres 16, a associação entre tabagismo e inserção no trabalho também varie segundo o gênero.

O objetivo do presente estudo foi investigar se a prevalência de tabagismo atual em homens e mulheres economicamente ativos está associada à inserção no mercado de trabalho, após ajuste por escolaridade, renda e situação de saúde. Discutem-se, ainda, hipóteses para explicar as associações encontradas.

\section{Métodos}

Este estudo utilizou dados da Pesquisa Nacional por Amostra de Domicílios (PNAD) realizada em 2008 pelo Instituto Brasileiro de Geografia e Estatísticas (IBGE). Anualmente, a PNAD obtém informações sobre características sociodemográficas, educacionais, de trabalho, entre outras. Em 2008, o suplemento de saúde introduzido nos anos de 1998 e 2003 incluiu comportamentos relacionados à saúde. A Pesquisa Especial de Tabagismo (PETab), agregada à PNAD 2008 e realizada em uma subamostra, investigou outros aspectos relacionados ao tabagismo além daqueles inseridos na PNAD 2008. Os questionários da PETab foram respondidos exclusivamente pelos próprios indivíduos, diferentemente da PNAD, que utiliza informante secundário 17.

Para essa análise, foram incluídos indivíduos com idade de 15 a 64 anos, que faziam parte da população economicamente ativa, residiam nas regiões metropolitanas de Fortaleza (Ceará), Recife (Pernambuco), Salvador (Bahia), Belo Horizonte (Minas Gerais), Rio de Janeiro, São Paulo, Curitiba (Paraná) e Porto Alegre (Rio Grande do Sul). Eles responderam às perguntas sobre tabagismo do suplemento de saúde da PNAD ou da PETab.

A PNAD utiliza uma amostra probabilística complexa obtida em dois ou três estágios de seleção. Foram incluídos no presente estudo apenas os municípios de regiões metropolitanas, onde a amostra é obtida em dois estágios. As unidades primárias de amostragem são os setores censitários e as unidades secundárias são os domicílios. 


\section{Variáveis estudadas}

Nesta pesquisa, a variável resposta foi o tabagismo atual, obtida por meio das perguntas: "Atualmente você fuma algum produto de tabaco?” $\mathrm{e}$ "Somando todos os cigarros que fumou na vida inteira, o total chega a, pelo menos, cinco maços ou cem cigarros?". A variável foi agrupada nas categorias: fumante (indivíduo que já havia fumado, pelo menos, cinco maços ou cem cigarros na vida e que fumava no momento da realização da pesquisa) e não fumante (indivíduo que não fumou, pelo menos, cinco maços ou cem cigarros, ou que já havia fumado, mas não fumava no momento da realização da pesquisa).

A variável explicativa de interesse foi situação no mercado de trabalho, categorizada em trabalho com proteção social, trabalho sem proteção social e desemprego. Trabalho com proteção social correspondeu ao trabalho com carteira assinada ou apenas com contribuição para previdência social. Trabalho sem proteção social foi aquele sem carteira assinada e sem contribuição para previdência social. Desemprego correspondeu à condição de indivíduos que não trabalhavam e procuraram por trabalho na semana de referência adotada pela PNAD.

As outras características sociodemográficas inseridas foram escolaridade, em anos completos de estudo (0-7, 8-10, 11-14, 15+), e renda domiciliar per capita, agrupada em quintis. A idade foi estudada por meio das seguintes faixas etárias: 15-24, 25-34, 35-44, 45-54, 55-64 anos. Incluímos três variáveis de saúde: relato de diagnóstico médico de asma ou bronquite (sim, não), relato de diagnóstico médico de doença do coração (sim, não) e de câncer (sim, não). Tais condições de saúde foram consideradas para ajuste porque são associadas à exposição ao tabaco e a piores condições de vida e de trabalho, sendo, portanto, potenciais fatores de confusão neste estudo.

\section{Análise}

Após a análise descritiva, foi estimada a prevalência de fumantes e o seu intervalo de $95 \%$ de confiança (IC95\%) em homens e mulheres; em seguida, estimou-se a prevalência de fumantes segundo as variáveis explicativas estudadas e o respectivo IC95\%. A associação entre a prevalência de fumantes e as variáveis explicativas foi medida por meio do teste de qui-quadrado de Pearson com nível de significância de 0,05. Em seguida, utilizando-se regressão de Poisson com variância robusta, foi feita a análise univariada para obter a razão de prevalência (RP) e seu IC95\%. Por último, realizou-se a análise multivariada com a inclusão das variáveis que se apre- sentaram associadas ao tabagismo com valor de $p \leq 0,20$. A categoria de referência para a análise foi não fumante.

A fim de corrigir as diferentes probabilidades de seleção de cada indivíduo, utilizaram-se pesos na estimativa das prevalências. A análise foi feita no programa Stata, versão 10.0 (Stata Corp., College Station, Estados Unidos), utilizando-se o procedimento svy (com fatores de ponderação), adequado para análises de dados obtidos por plano amostral complexo.

O presente estudo utiliza dados de inquérito populacional e foi analisado de acordo com os princípios da Declaração de Helsinki.

\section{Resultados}

Foram estudados 62.471 indivíduos com média de idade de 36,2 anos; $54 \%$ eram do sexo masculino, sem diferença na média de idade entre os sexos. A prevalência total de fumantes foi 18,1 , variando de $20,8 \%$ entre homens a $14,9 \%$ entre mulheres. As características da população estudada estratificada por sexo são mostradas na Tabela 1 . Dos homens, 30,3\% estavam inseridos no trabalho sem proteção social e $7 \%$, desempregados; entre as mulheres, esses percentuais foram respectivamente $34,5 \%$ e $12,2 \%$.

A prevalência de fumantes no sexo masculino foi mais elevada entre os homens que trabalhavam sem proteção social; aumentou com a idade até a faixa de 45-54 anos, sofrendo leve declínio entre os tinham de 55-64 anos; diminuiu inversamente com a escolaridade e a renda domiciliar per capita. Entre as mulheres, a prevalência de tabagismo foi mais elevada naquelas inseridas no trabalho sem proteção social; aumentou com a idade, atingindo o maior percentual entre as que tinham 45-54 anos; diminuiu com o aumento da escolaridade e da renda. As fumantes relataram asma e doença do coração com maior frequência do que as não fumantes (Tabela 2).

$\mathrm{Na}$ análise univariada, entre os homens, ser fumante mostrou-se positivamente associado ao trabalho sem proteção social, ao desemprego, à idade, ao relato de doença do coração e de câncer, e negativamente associado ao aumento da escolaridade e da renda domiciliar per capita. Entre as mulheres, o tabagismo esteve positivamente associado com inserção em ocupações sem proteção social, idade, relato de asma e/ou bronquite e de doença do coração, e negativamente associado à escolaridade e à renda domiciliar per capita (Tabela 2).

Associações independentes entre a situação no mercado de trabalho e o tabagismo são apresentadas na Tabela 3. No sexo masculino, após 
Distribuição da população, segundo características sociodemográficas e variáveis de saúde estudadas. Regiões metropolitanas brasileiras, Pesquisa Nacional por Amostra de Domicílios, 2008 (PNAD 2008).

\begin{tabular}{|c|c|c|c|}
\hline & Homens (\%) & & Mulheres (\%) \\
\hline \multicolumn{4}{|l|}{ Características sociodemográficas } \\
\hline \multicolumn{4}{|l|}{ Idade (anos) } \\
\hline $15-24$ & 20,6 & & 20,3 \\
\hline $25-34$ & 27,8 & & 28,2 \\
\hline $35-44$ & 23,7 & & 24,5 \\
\hline $45-54$ & 18,8 & & 18,8 \\
\hline \multirow[t]{2}{*}{$55-64$} & 9,0 & & 8,1 \\
\hline & & $p=0,0059$ & \\
\hline \multicolumn{4}{|l|}{ Situação no mercado de trabalho } \\
\hline Trabalho com proteção social & 62,8 & & 53,4 \\
\hline Trabalho sem proteção social & 30,3 & & 34,5 \\
\hline \multirow[t]{2}{*}{ Desempregado } & 7,0 & & 12,2 \\
\hline & & $p<0,0001$ & \\
\hline \multicolumn{4}{|l|}{ Escolaridade (anos de estudo) } \\
\hline $0-7$ & 29,2 & & 24,2 \\
\hline $8-10$ & 20,2 & & 18,1 \\
\hline $11-14$ & 38,9 & & 42,4 \\
\hline \multirow[t]{2}{*}{$15+$} & 11,6 & & 15,3 \\
\hline & & $p<0,0001$ & \\
\hline \multicolumn{4}{|l|}{ Renda domiciliar per capita } \\
\hline 1ㅇ quintil (menor) & 16,6 & & 16,5 \\
\hline 2o quintil & 18,7 & & 19,0 \\
\hline 3o quintil & 20,5 & & 20,5 \\
\hline 4o quintil & 22,4 & & 21,5 \\
\hline \multirow[t]{2}{*}{ 5o quintil (maior) } & 21,8 & & 22,5 \\
\hline & & $p=0,1570$ & \\
\hline \multicolumn{4}{|l|}{ Características de saúde } \\
\hline \multicolumn{4}{|l|}{ Asma } \\
\hline Não & 96,6 & & 95,1 \\
\hline \multirow[t]{2}{*}{ Sim } & 3,4 & & 4,9 \\
\hline & & $p<0,0001$ & \\
\hline \multicolumn{4}{|l|}{ Doença do coração } \\
\hline Não & 97,4 & & 96,9 \\
\hline \multirow[t]{2}{*}{ Sim } & 2,6 & & 3,1 \\
\hline & & $p=0,0008$ & \\
\hline \multicolumn{4}{|l|}{ Câncer } \\
\hline Não & 99,7 & & 99,4 \\
\hline \multirow[t]{2}{*}{ Sim } & 0,3 & & 0,6 \\
\hline & & $p<0,0001$ & \\
\hline
\end{tabular}

ajuste pela idade, pelas demais características sociodemográficas e pelas variáveis de saúde, a associação entre tabagismo e inserção no trabalho sem proteção social foi atenuada, mas ainda houve frequência $31 \%$ maior de fumantes entre os que se encontravam nessa situação do que entre os que trabalhavam com proteção social. O per- centual de fumantes entre desempregados também foi $31 \%$ maior do que entre os homens inseridos no trabalho com proteção social. Apenas a escolaridade e a idade se mantiveram associadas ao tabagismo. Entre as mulheres, a associação entre trabalho sem proteção social e tabagismo foi mantida, embora com menor magnitude; de- 
Prevalência de tabagismo (\%) e razão de prevalências (RP), de acordo com as características sociodemográficas e variáveis de saúde estudadas em homens e mulheres. Regiões metropolitanas brasileiras, Pesquisa Nacional por Amostra de Domicílios (PNAD 2008).

\begin{tabular}{|c|c|c|c|c|}
\hline & \multicolumn{2}{|c|}{ Prevalência (IC95\%) } & \multicolumn{2}{|c|}{ RP (IC95\%) } \\
\hline & Homens & Mulheres & Homens & Mulheres \\
\hline \multicolumn{5}{|l|}{ Características sociodemográficas } \\
\hline \multicolumn{5}{|l|}{ Idade (anos) } \\
\hline $15-24$ & $14,1(13,2-15,3)$ & $8,9(8,0-9,9)$ & 1,00 & 1,00 \\
\hline $25-34$ & $19,5(18,5-20,5)$ & $11,9(11,1-12,8)$ & $1,38(1,27-1,50)$ & $1,33(1,18-1,51)$ \\
\hline $35-44$ & $22,3(21,2-23,4)$ & $17,2(16,2-18,3)$ & $1,58(1,45-1,72)$ & $1,93(1,71-2,18)$ \\
\hline $45-54$ & $26,0(24,7-27,3)$ & $23,0(21,6-24,3)$ & $1,84(1,69-2,00)$ & $2,58(2,29-2,90)$ \\
\hline $55-64$ & $25,5(23,7-27,4)$ & $15,2(13,6-17,1)$ & $1,80(1,63-1,99)$ & $1,71(1,47-2,00)$ \\
\hline \multicolumn{5}{|l|}{ Situação no mercado de trabalho } \\
\hline Trabalho com proteção social & $18,3(17,4-18,7)$ & $12,8(12,2-13,5)$ & 1,00 & 1,00 \\
\hline Trabalho sem proteção social & $26,4(25,4-27,4)$ & $18,5(17,6-19,4)$ & $1,47(1,39-1,54)$ & $1,44(1,35-1,55)$ \\
\hline Desempregado & $20,8(20,3-21,4)$ & $14,9(12,8-15,7)$ & $1,20(1,09-1,32)$ & $1,11(0,99-1,24)$ \\
\hline \multicolumn{5}{|l|}{ Escolaridade (anos de estudo) } \\
\hline $0-7$ & $30,0(29,0-31,1)$ & $22,4(21,2-23,5)$ & 1,00 & 1,00 \\
\hline $8-10$ & $21,8(20,7-23,0)$ & $16,2(15,0-17,5)$ & $0,73(0,68-0,76)$ & $0,72(0,66-0,79)$ \\
\hline $11-14$ & $15,4(14,6-16,2)$ & $11,8(11,1-12,5)$ & $0,51(0,48-0,54)$ & $0,53(0,49-0,57)$ \\
\hline $15+$ & $14,6(13,7-16,1)$ & $10,4(9,3-11,6)$ & $0,48(0,44-0,54)$ & $0,46(0,41-0,52)$ \\
\hline \multicolumn{5}{|l|}{ Renda domiciliar per capita } \\
\hline 1ㅇ quintil (menor) & $25,5(24,3-26,8)$ & $18,7(17,5-20,1)$ & 1,00 & 1,00 \\
\hline 2o quintil & $22,2(21,0-23,5)$ & $16,1(14,9-17,3)$ & $0,87(0,81-0,94)$ & $0,86(0,76-0,95)$ \\
\hline 3o quintil & $22,0(20,7-23,2)$ & $14,7(13,7-15,9)$ & $0,86(0,80-0,93)$ & $0,79(0,71-0,87)$ \\
\hline 4o quintil & $19,5(18,4-20,7)$ & $13,8(12,8-15,0)$ & $0,76(0,71-0,82)$ & $0,74(0,67-0,82)$ \\
\hline $5 \circ$ quintil (maior) & $17,0(15,9-18,1)$ & $12,4(11,4-13,4)$ & $0,66(0,61-0,72)$ & $0,66(0,59-0,74)$ \\
\hline \multicolumn{5}{|l|}{ Características de saúde } \\
\hline \multicolumn{5}{|l|}{ Asma/bronquite } \\
\hline Não & $20,8(20,3-21,4)$ & $14,7(14,2-15,2)$ & 1,00 & 1,00 \\
\hline Sim & $20,0(17,4-23,0)$ & $19,2(16,8-21,8)$ & $0,96(0,83-1,11)$ & $1,30(1,14-1,80)$ \\
\hline \multicolumn{5}{|l|}{ Doença do coração } \\
\hline Não & $20,8(20,3-21,3)$ & $14,8(14,2-15,2)$ & 1,00 & 1,00 \\
\hline Sim & $22,0(18,8-25,5)$ & $22,7(19,6-26,2)$ & $1,06(0,91-1,23)$ & $1,55(1,33-1,80)$ \\
\hline \multicolumn{5}{|l|}{ Câncer } \\
\hline Não & $20,8(20,3-21,3)$ & $14,9(14,4-15,4)$ & 1,00 & 1,00 \\
\hline Sim & $27,2(18,5-38,1)$ & $16,6(10,7-15,4)$ & $1,31(0,91-1,88)$ & $1,11(0,73-1,69)$ \\
\hline
\end{tabular}

semprego tornou-se positivamente associado ao tabagismo. Foram mantidas as associações com idade, escolaridade, relato de asma e/ou bronquite e de doença do coração. A renda domiciliar per capita permaneceu negativamente associada à condição de fumante apenas entre as mulheres que se encontravam no terceiro quintil de renda (Tabela 3).

\section{Discussão}

Nossos resultados confirmam que a situação no mercado de trabalho é uma dimensão independente das desigualdades sociais em saúde, especificamente das desigualdades sociais e tabagismo. Estar inserido no trabalho sem proteção social e estar desempregado associaram-se à maior prevalência de tabagismo entre homens e entre mulheres, independentemente da escolaridade, da renda e da presença ou não de problemas de saúde diretamente relacionados ao tabagismo. 
Características sociodemográficas e variáveis de saúde associadas com tabagismo. Regiões metropolitanas brasileiras,

Pesquisa Nacional por Amostra de Domicílios (PNAD 2008).

\begin{tabular}{|c|c|c|}
\hline & \multicolumn{2}{|c|}{ RP ajustada (IC95\%) } \\
\hline & Homens & Mulheres \\
\hline \multicolumn{3}{|l|}{ Características sociodemográficas } \\
\hline \multicolumn{3}{|l|}{ Idade (anos) } \\
\hline $15-24$ & 1,00 & 1,00 \\
\hline $25-34$ & $1,50(1,38-1,64)$ & $1,43(1,25-1,63)$ \\
\hline $35-44$ & $1,63(1,49-1,77)$ & $1,95(1,71-2,22)$ \\
\hline $45-54$ & $1,84(1,69-2,01)$ & $2,46(2,16-2,81)$ \\
\hline $55-64$ & $1,67(1,50-1,86)$ & $1,54(1,30-1,83)$ \\
\hline \multicolumn{3}{|l|}{ Situação no mercado de trabalho } \\
\hline Trabalho com proteção social & 1,00 & 1,00 \\
\hline Trabalho sem proteção social & $1,31(1,24-1,38)$ & $1,22(1,13-1,31)$ \\
\hline Desempregado & $1,31(1,18-1,44)$ & $1,16(1,03-1,32)$ \\
\hline \multicolumn{3}{|l|}{ Escolaridade (anos de estudo) } \\
\hline $0-7$ & 1,00 & 1,00 \\
\hline $8-10$ & $0,82(0,77-0,89)$ & $0,88(0,80-0,97)$ \\
\hline $11-14$ & $0,59(0,56-0,63)$ & $0,68(0,62-0,75)$ \\
\hline $15+$ & $0,52(0,48-0,58)$ & $0,54(0,47-0,63)$ \\
\hline \multicolumn{3}{|l|}{ Renda domiciliar per capita } \\
\hline 1ㅇ quintil (menor) & - & 1,00 \\
\hline 2o quintil & - & $0,93(0,84-1,03)$ \\
\hline 3o quintil & - & $0,89(0,80-0,99)$ \\
\hline 4의 quintil & - & $0,89(0,80-1,00)$ \\
\hline 5o quintil (maior) & - & $0,91(0,80-1,03)$ \\
\hline \multicolumn{3}{|l|}{ Características de saúde } \\
\hline \multicolumn{3}{|l|}{ Asma } \\
\hline Não & - & 1,00 \\
\hline Sim & - & $1,29(1,12-1,48)$ \\
\hline \multicolumn{3}{|l|}{ Doença do coração } \\
\hline Não & - & 1,00 \\
\hline Sim & - & $1,26(1,08-1,48)$ \\
\hline
\end{tabular}

Os resultados mostraram ainda que as prevalências de tabagismo na população economicamente ativa foram mais elevadas nos homens inseridos em trabalhos sem proteção social.

O trabalho pode influenciar diretamente a ocorrência de um evento de saúde ou indiretamente por meio dos comportamentos relacionados à saúde, já que a exposição aumenta o risco de adoecimento ao longo da vida. O desemprego influencia negativamente eventos de saúde, tanto subjetivos, quanto objetivos 18,19,20,21. É possível que essa relação seja potencializada por comportamentos de risco relacionados à saúde, uma vez que indivíduos desempregados estão mais vulneráveis a esses comportamentos do que os empregados 22 .
Nossos resultados corroboram os estudos que mostram o desemprego positivamente associado ao tabagismo 23,24 , associação que se torna ainda mais intensa quanto maior a duração de desemprego ${ }^{25}$. Ao estudar trajetórias no mercado de trabalho (emprego estável até desemprego de longa duração) e mudanças na situação de fumante/não fumante ao longo de cinco anos, Virtanem et al. 26 verificaram que os homens que se encontravam desempregados na linha de base e no seguimento fumavam mais cigarros/dia do que os que trabalhavam. Entre as mulheres, aquelas desempregadas nos dois períodos apresentaram tendência de incremento no número de cigarros fumados por dia $(\mathrm{p}=$ 0,054). 
A influência das formas precárias de inserção no mercado de trabalho sobre eventos de saúde, como transtornos mentais 27,28 , percepção da saúde 29 e relato de doenças crônicas 30 , são descritos na literatura. Sua relação com comportamentos de risco à saúde é menos conhecida, e os resultados, pouco consistentes. Análise de coorte de 4.851 trabalhadores finlandeses não identificou diferenças em relação aos comportamentos de saúde e inserção em formas de trabalho mais ou menos estáveis no período de 1997 a 2000 31. Estudo com base em outra coorte de trabalhadores finlandeses, esta acompanhada por cinco anos, também não verificou associação entre tabagismo e trajetória dos indivíduos para situações atípicas de trabalho, assim como não houve diferença na probabilidade de parar de fumar 26. Embora não seja uma medida direta de precariedade no trabalho, mudança frequente de emprego está muito relacionada com ocupações instáveis e está associada a comportamentos não saudáveis, inclusive ao fumo ${ }^{32}$. Um estudo realizado na Coréia do Sul 33 observou que trabalhadores inseridos em formas precárias de trabalho apresentavam pior perfil de comportamentos de risco para saúde, resultado semelhante aos identificados na presente análise.

Se, por um lado, nossos resultados indicam maior segregação das mulheres para as formas de trabalho precário e para o desemprego, a magnitude das associações do presente estudo sugere gradiente social para o tabagismo mais acentuado nos homens. A magnitude da associação entre a situação no mercado de trabalho e fumo foi mais elevada no sexo masculino. Poucos estudos que investigaram a relação entre ocupação precária, desemprego e saúde consideraram os diferenciais por gênero. Resultados similares foram descritos para ambos os sexos 34 , assim como variações similares às mostradas pelos nossos resultados. Castro et al. 35 identificaram diferença no gradiente social para o tabagismo entre homens e mulheres. No que se refere aos trabalhadores de menor qualificação e desempregados, em amostra representativa da população americana acima de 18 anos de idade, as prevalências foram mais elevadas no sexo masculino do que no feminino. A tendência de associação inversa entre escolaridade e risco de se tornar fumante foi mais modesta entre as mulheres quando comparadas aos homens, em análise longitudinal 36. Artacoz et al. 37, ao discutirem gênero, saúde e trabalho, mais especificamente o desemprego, creditam aos papéis socialmente atribuídos aos homens e às mulheres uma parte da explicação das diferenças observadas. Segundo os autores, para as mulheres, viver com cônjuge pode resultar em suporte financeiro e emocional, minimizando os efeitos do trabalho precário ou desemprego sobre a saúde e comportamentos relacionados; por outro lado, para os homens, essa mesma situação pode causar efeito reverso. Há ainda a possibilidade de, diante da perda do trabalho, as mulheres assumirem mais intensamente outros papéis, como as responsabilidades domésticas e com os filhos, atenuando, de certa forma, essas associações. Infelizmente, não possuímos informações referentes ao estado civil e atividades domésticas para averiguar sua influência em nossos resultados.

A relação entre situação desfavorável no mercado de trabalho e maior vulnerabilidade a comportamentos prejudiciais à saúde é provavelmente mediada pela privação material e por fatores psicossociais 38. Restrição financeira, além dos aspectos estritamente a ela relacionados, ou seja, menor recurso para atender necessidades, também reforça o impacto dos fatores psicossociais, como percepção de perda de controle, baixa autoestima, perda de papel social e de perspectiva. Nessas condições, fumar, ainda que represente um gasto não "essencial”, pode auxiliar o indivíduo a lidar com problemas objetivos e subjetivos, atuando como redutor da ansiedade e da tensão ${ }^{32}$. Altos níveis de pressão e eventos estressantes da vida já foram associados positivamente ao tabagismo e à maior intensidade deste ${ }^{39}$. A exclusão social também está presente nas formas de trabalho precárias, pois situações ou percepções de instabilidade, ausência de perspectiva no trabalho, longas jornadas ou jornadas muito restritas de trabalho, podem levar a insatisfações, favorecendo a adoção de comportamentos que reduzem momentaneamente as tensões, como o tabagismo e o álcool, entre outros. Condições psicossociais adversas no ambiente de trabalho foram positivamente relacionadas ao tabagismo em estudos já realizados 40,41 .

Outro aspecto que pode influenciar a menor frequência de tabagismo entre trabalhadores com proteção social, quando comparados aos em situação inversa, são as restrições ao fumo no ambiente de trabalho. É possível que, assim, forme-se um ambiente favorável à cessação do tabagismo. No Brasil, existe uma série de incentivos para o fim desse comportamento, que compreendem, inclusive, o estabelecimento dos ambientes livres de tabaco. Mas essas ações estão mais presentes em ambientes públicos de trabalho e setores formais da economia.

A inserção no mercado de trabalho captura uma dimensão da posição socioeconômica dos indivíduos na vida adulta e está relacionada com a escolaridade e a renda. Em nossa análise, a escolaridade também influenciou o tabagismo 
em ambos os sexos. Já a renda, após o ajuste, apresentou pouca importância nesses resultados. Conforme observado na análise univariada, é razoável supor que o efeito da renda sobre o tabagismo seja mediado pela escolaridade e pela situação no mercado de trabalho. Tendo em vista que fumar, assim como parar de fumar, é um comportamento social e culturalmente determinado, compreende-se que o trabalho, na qualidade de expressão de engajamento social, seja mais relevante que a renda em si para o ato de fumar/deixar de fumar, uma vez considerada a escolaridade 42 .

As doenças investigadas, respiratórias, cardíacas e câncer, podem ser causadas ou agravadas pelo tabagismo, sendo motivos frequentes de interrupção desse hábito. No presente trabalho, a relação entre as variáveis de saúde estudadas e o tabagismo só foi observada entre as mulheres. Como esta análise se restringe à população economicamente ativa, os muito velhos e muito doentes foram excluídos; além disso, sendo a análise restrita ao tabagismo atual, a ausência de associação entre doença e tabagismo não surpreende. Em estudos com todos os adultos, e não apenas os economicamente ativos, tais associações são percebidas, especialmente entre os mais velhos e os ex-fumantes 43,44

A menor prevalência de tabagismo entre os indivíduos mais jovens sugere um efeito de coorte, expressando a tendência de queda das prevalências no Brasil nas últimas décadas. Se a menor prevalência em jovens sugere redução da exposição ao tabaco, em adultos mais velhos sugere a interrupção do hábito de fumar. Tais efeitos expressam o resultado dos esforços de intervenções de saúde pública no país, voltados não só para a prevenção, como também para a redução do tabagismo 11 .

Os resultados desta pesquisa devem ser interpretados com cuidado, uma vez que se trata de um estudo transversal, não sendo possível estabelecer relação temporal entre situação no mercado de trabalho e tabagismo. Outro aspecto a ser considerado é que o tabagismo pode reduzir as oportunidades de trabalho com proteção social, influenciando nossos resultados. Infelizmente, não dispomos de dados sobre problemas de saúde no período anterior a esta investigação, mas vale ressaltar que o relato de tabagismo na vida (dado não mostrado) e de tabagismo atual foram mais frequentes entre os trabalhadores sem proteção social. É preciso considerar também que, em relação a $54,6 \%$ dos participantes do sexo masculino e a $36,8 \%$ dos do sexo feminino, os questionários foram respondidos por informantes secundários. No entanto, a direção das associações não foi modificada quando realizamos análises separadas segundo o tipo de respondente. Ademais, informações de saúde mais objetivas, como o tabagismo, estão menos sujeitas a erros de informação em estudos que utilizam informantes secundários 45.

O suplemento de saúde da PNAD tem possibilitado ampla produção de estudos acerca das condições de saúde 46 , do acesso a serviços de saúde e utilização destes 47 , das desigualdades sociais em saúde 48 , inclusive daquelas relacionadas à inserção no mercado de trabalho ${ }^{30}$. A inclusão de variáveis comportamentais de saúde na PNAD 2008 permitiu investigar se a inserção no mercado de trabalho também está associada ao tabagismo na população metropolitana do país. Nossos resultados mostram que o hábito de fumar é mais frequente entre desempregados e trabalhadores informais, especialmente do sexo masculino. Como a iniciação ao uso do tabaco, bem como de outras drogas, tende a se estabelecer durante a adolescência 1, antecedendo, portanto, o ingresso no mercado de trabalho, é bastante provável que o trabalho contribua de forma diferente para a consolidação do comportamento entre os iniciados. Por um lado, pode atuar como um promotor, em maior ou menor escala, da cessação do tabagismo em locais que apresentam restrições diretas ao fumo no ambiente de trabalho, o que geralmente ocorre no emprego formal. Por outro lado, entre os empregados sem vínculo social, o comportamento de fumar sofreria mais influência da convivência com os pares, sem mediação externa.

Políticas que favoreçam o crescimento do trabalho com proteção social e assegurem ações de promoção da saúde no trabalho têm papel primordial na qualidade de vida e saúde da população adulta. 


\section{Resumo}

O presente estudo teve como objetivo verificar se o tabagismo atual está associado à inserção no mercado de trabalho, após ajuste por escolaridade, renda e saúde em homens e mulheres; objetivou, também, discutir hipóteses explicativas. Foram estudados indivíduos de 15 a 64 anos, residentes em regiões metropolitanas brasileiras, pertencentes à população economicamente ativa, incluídos na Pesquisa Nacional por Amostra de Domicílios (2008). Razão de prevalência (RP) e intervalo de 95\% de confiança (IC95\%) foram obtidos pela regressão de Poisson. Após ajustes, o tabagismo foi estatisticamente maior em indivíduos inseridos no trabalho sem proteção social (homens, $R P=1,31$, IC95\%: 1,24-1,38; mulheres, $R P=1,22$, IC95\%: 1,121,31) e desempregados (homens, $R P=1,31$, IC95\%: 1,18-1,44; mulheres, $R P=1,16$, IC95\%: 1,03-1,32). Prevalências mais altas de tabagismo foram observadas nos homens inseridos no trabalho sem proteção social. Nossos resultados confirmam que a situação no mercado de trabalho é uma dimensão independente das desigualdades sociais no tabagismo. O gradiente social observado foi mais acentuado nos homens, mas a associação entre inserção no trabalho e tabagismo está presente nos dois gêneros.

Tabagismo; Mercado de Trabalho; Desigualdades em Saúde

\section{Colaboradores}

L. Giatti e S. M. Barreto trabalharam na concepção, delineamento, análise e redação do artigo.

\section{Agradecimentos}

L. Giatti é bolsista do Programa de Apoio a Projetos Institucionais com a Participação de Recém-Doutores, da Coordenação de Aperfeiçoamento de Pessoal de Nível Superior (PRODOC-CAPES). S. M. Barreto é bolsista de produtividade em pesquisa do Conselho Nacional de Desenvolvimento Científico e Tecnológico (CNPq; processo $n^{\circ}$. 300159/99-4).

\section{Referências}

1. Wünsch-Filho V, Mirra AP, López RVM, Antunes LF. Tabagismo e câncer no Brasil: evidências e perspectivas. Rev Bras Epidemiol 2010; 13:175-87.

2. World Health Organization. Tobacco. (Fact Sheet, 339). http://www.who.int/mediacentre/factsheets/ fs339/en/index.html (acessado em 10/Ago/2010).

3. Haustein KO. Smoking and poverty. Eur J Cardiovasc Prev Rehabil 2006; 13:312-8.

4. Sorensen G, Barbeau E, Hunt MK, Emmons K. Reducing social disparities in tobacco use: a socialcontextual model for reducing tobacco use among blue-collor workers. Am J Public Health 2004; 94:230-9.

5. Karvonen S, Sipilä P, Martikainen P, Rahkonen O, Laaksonen M. Smoking in context - a multilevel approach to smoking among females in Helsinki. BMC Public Health 2008; 8:134.

6. Baumann M, Spitz E, Guillermin F, Ravaud JF, Choquet M, Falissard B, et al. Associations of social and material deprivation with tobacco, alcohol, and psychotropic drug use, and gender: a populationbased study. Int J Health Geogr 2007; 6:50.
7. Peretti-Watel P, Constance J, Seror V, Beck F. Cigarettes and social differentiation in France: is tobacco use increasingly concentrated among the poor? Addiction 2009; 10:1718-28.

8. Tramacere I, Gallus S, Zuccaro P, Colombo P, Rossi S, Boffetta P, et al. Socio-demographic variation in smoking habits: Italy, 2008. Prev Med 2009; 48:213-7.

9. Barbeau EM, Krieger N, Soobader M. Working class matters: socioeconomics disadvantage, race/ ethinicity, gender and smoking in NHIS 2000. Am J Public Health 2004; 94:269-78.

10. Najman JM, Toloo G, Siskind V. Socioeconomic disadvantage and changes in health risk behaviours in Australia: 1989-90 to 2001. Bull World Health Organ 2006; 84:976-84.

11. Monteiro CA, Cavalcante TM, Moura EC, Claro RM, Szwarcwald CL. Population-based evidence of a strong decline in the prevalence of smokers in Brazil (1989-2003). Bull World Health Organ 2007; 85:527-34. 
12. Instituto Nacional de Câncer. Inquérito domiciliar sobre comportamentos de risco e morbidade referida de doenças e agravos não transmissíveis: Brasil, 15 capitais e Distrito Federal, 2002-2003. Rio de Janeiro: Instituto Nacional de Câncer; 2004.

13. Silva GA, Valente JG, Almeida LM, Moura EC, Malta DC. Tabagismo e escolaridade no Brasil, 2006. Rev Saúde Pública 2009; 43:48-56.

14. Clougherty JE, Souza K, Cullen MR. Work and its role in shaping the social gradient in health. Ann $\mathrm{N}$ Y Acad Sci 2010; 1186:102-24.

15. Benach J, Muntaner C, Santana V. Employment conditions and health inequalities. Final report to the WHO Commission on Social Determinants of Health. http://www.who.int/social_determinants/ resources/articles/emconet_who_report.pdf/ (acessado em 05/Ago/2010).

16. Weden MM, Astone NM, Bishai D. Racial, ethnic, and gender differences in smoking cessation associated with employment and joblessness through young adulthood in the US. Soc Sci Med 2006; 62:303-16.

17. Instituto Brasileiro de Geografia e Estatística. Pesquisa Nacional por Amostra de Domicílios: síntese de indicadores 2008. http://www.ibge.gov.br/ho me/estatistica/populacao/trabalhoerendimento/ pnad2008/notas_sintese.pdf(acessado em20/Ago/ 2010).

18. Talala K, Huurre T, Aro H, Martelin T, Praatta R. Trends in socio-economic differences in self-reported depression during the years 1979-2002 in Finland. Soc Psychiatry Psychiatr Epidemiol 2009; 44:871-9.

19. Giatti L, Barreto SM, César CC. Unemployment and self-rated health: neighborhood influence. Soc Sci Med 2010; 71:815-23.

20. Szwarcwald CL, Bastos FI, Esteves MAP. State of animus among Brazilians: influence of socioeconomic context? Cad Saúde Pública 2005; 21 Suppl: S33-42.

21. Voss M, Nylén L, Floderus B, Diderichsen F, Terry $\mathrm{RD}$. Unemployment and early cause-specific mortality: a study based on the Swedish Twin Registry. Am J Public Health 2004; 94:2155-61.

22. Vogli R, Santinello M. Unemployment and smoking: does psychosocial stress matter? Tob Control 2005; 14:389-95.

23. Hammarstrom A, Janlert U. Early unemployment can contribute to adult health problems: results from a longitudinal study of school leavers. J Epidemiol Community Health 2002; 56:624-30.

24. Hammarstrom A, Janlert U. Unemployment: an important predictor for future smoking: a 14-year follow up study of school leavers. Scand J Public Health 2003; 31:229-32.

25. Schunck R, Rogger BG. Unemployment and its association with health-relevant actions: investigating the role of time perspective with German census data. Int J Public Health 2010; 55:271-8.

26. Virtanen P, Vahtera J, Broms U, Sillanmäki L, Kivimäki M, Koskenvuo M. Employment trajectory as determinant of change in health-related lifestyle: the prospective HeSSup study. Eur J Public Health 2008; 18:504-8.
27. Ludemir AB, Lewis G. Informal work and common mental disorders. Soc Psychiatr Epidemiol 2003; 38:485-9.

28. Reine I, Novo M, Hammarström A. Does transition from an unstable labour market position to permanent employment protect mental health? Results from a 14-year follow-up of school-leavers. BMC Public Health 2008; 8:159.

29. Giatti L, Barreto SM, César CC. Household context and self-rated health: the effect of unemployment and informal work. J Epidemiol Community Health 2008; 62:1079-85.

30. Giatti L, Barreto SM, César CC. Informal work, unemployment and health in Brazilian metropolitan areas, 1998 and 2003. Cad Saúde Pública 2008; 24:2396-406.

31. Virtanen M, Kivimaki M, Eloviano M, Vahtera J, Ferrie JE. From insecure to secure employment: change in work, health, health related behaviours, and sickness absence. Occup Environ Med 2003; 60:948-53.

32. Metcalfe C, Smith GD, Sterne JAC, Heslop P, Macleod J, Hart C. Frequent job change and associated health. Soc Sci Med 2003; 56:1-15.

33. Kim H, Khang YH, Muntaner C, Chun H, Cho SI. Gender, precarious work, and chronic diseases in South Korea. Am J Ind Med 2008; 51:748-57.

34. Virtanen M, Kivimaki M, Joensuu M, Virtanen P, Elovainio M, Vahtera J. Temporary employment and health: a review. Int J Epidemiol 2005; 34: 610-22.

35. Castro AB, Garcia G, Gee GC, Hsin-Chun Tsai J, Rue T, Takeuchi DT. Smoking and the Asian American workforce in the National Latino and Asian American Study. Am J Ind Med 2010; 53:171-8.

36. Johnson EO, Novak SP. Onset and persistence of daily smoking: the interplay of socioeconomic status, gender, and psychiatric disorders. Drug Alcohol Depend 2009; 104 Suppl 1:S50-7.

37. Artacoz L, Benach J, Borrell C, Cortés I. Unemployment and mental health: understanding the interactions among gender, family roles and social class. Am J Public Heatlh 2004; 94:82-8.

38. Bjarnason T, Sigurdardottir SJ. Psychological distress during unemployment and beyond: social support and material deprivation among youth in six northern European countries. Soc Sci Med 2003; 56:973-85.

39. Metcalfe C, Smith GD, Wadsworth E, Sterne JAC, Heslop P, Macleod J, et al. A contemporary validation of the Reeder Stress Inventory. Br J Health Psychol 2003; 8:83-94.

40. Radi S, Ostry A, LaMontagne AD. Job stress and other working conditions: relationships with smoking behaviors in a representative sample of working Australians. Am J Ind Med 2007; 50: 584-96.

41. Lalluka T, Lahelma E, Rahkonen O, Ross E, Laaksonem E, Martikainen P, et al. Associations of job strain and working overtime with adverse health behaviors and obesity: evidence from the Whitehall II Study, Helsinki Health Study and the Japanese Civil Servants Study. Soc Sci Med 2008; 66:1681-98. 
42. Sorensen G, Emmons K, Stoddard AM, Linnan L, Avrunin J. Do social influences contribute to occupational differences in quitting smoking and attitudes toward quitting? Am J Health Promot 2002; 16:135-41.

43. Sachs-Ericsson N, Schmidt NB, Zvolensky JM, Mitchell M, Collins N, Blazer DG. Smoking cessation behavior in older adults by race and gender: the role of health problems and psychological distress. Nicotine Tob Res 2009; 11:433-43.

44. Twardella D, Loew M, Rothenbacher D, Stegmaier C, Ziegler H, Brenner $\mathrm{H}$. The diagnosis of a smoking-related disease is a prominent trigger for smoking cessation in a retrospective cohort study. J Clin Epidemiol 2006; 59:82-9.

45. Jardim R, Barreto SM, Giatti L. Confiabilidade das informações obtidas de informante secundário em inquéritos populacionais. Cad Saúde Pública 2010; 26:1537-48.
46. Barros MBA, César CLG, Carandina L, Torre GD. Desigualdades sociais na prevalência de doenças crônicas no Brasil, PNAD-2003 Ciênc Saúde Coletiva 2006; 11:911-26.

47. Travassos C, Oliveira EXG, Viacava F. Desigualdades geográficas e sociais no acesso aos serviços de saúde no Brasil: 1998 e 2003. Ciênc Saúde Coletiva 2006; 11:975-86.

48. Barata RB, Almeida MF, Monteiro CV, Silva ZP. Gender and health inequalities among adolescents and adults in Brazil, 1998. Rev Panam Salud Pública 2007; 21:320-7.

Recebido em 27/Out/2010

Versão final reapresentada em 27/Fev/2011

Aprovado em 18/Mar/2011 